\title{
Critical slowing-down and field-dependent paramagnetic fluctuations in the skyrmion host EuPtSi: $\mu$ SR and NMR studies
}

\author{
N. Higa $\odot,{ }^{1,}{ }^{*}$ T. U. Ito $\odot,{ }^{1}$ M. Yogi $\odot,{ }^{2}$ T. Hattori, ${ }^{1}$ H. Sakai, ${ }^{1}$ S. Kambe $\odot,{ }^{1}$ Z. Guguchia, ${ }^{3}$ W. Higemoto, ${ }^{1}$ \\ M. Nakashima $₫,{ }^{4}$ Y. Homma, ${ }^{5}$ A. Nakamura, ${ }^{5}$ F. Honda,${ }^{5}$ Y. Shimizu $\odot,{ }^{5}$ D. Aoki, ${ }^{5}$ M. Kakihana, ${ }^{2}$ M. Hedo, ${ }^{2}$ T. Nakama, ${ }^{2}$ \\ Y. Ōnuki, ${ }^{2}$ and Y. Tokunaga ${ }^{1}$ \\ ${ }^{1}$ Advanced Science Research Center, Japan Atomic Energy Agency, Tokai-mura, Ibaraki 319-1195, Japan \\ ${ }^{2}$ Department of Physics and Earth Sciences, Faculty of Science, University of the Ryukyus, Okinawa 903-0213, Japan \\ ${ }^{3}$ Laboratory for Muon-Spin Spectroscopy, Paul Scherrer Institut, CH-5232 Villigen PSI, Switzerland \\ ${ }^{4}$ Faculty of Science, Shinshu University, Matsumoto, Nagano 390-8621, Japan \\ ${ }^{5}$ Institute for Materials Research, Tohoku University, Oarai, Ibaraki 311-1313, Japan
}

(Received 2 November 2020; revised 11 May 2021; accepted 30 June 2021; published 27 July 2021)

\begin{abstract}
We report the results of muon spin relaxation $(\mu \mathrm{SR})$ and ${ }^{29} \mathrm{Si}$ NMR measurements carried out on the cubic chiral magnet EuPtSi. This compound exhibits a helimagnetic transition at $T_{\mathrm{N}}=4 \mathrm{~K}$, and hosts a unique skyrmion phase characterized by a short modulation period and strong anisotropy under magnetic fields. Our zero-field $\mu \mathrm{SR}$ experiments revealed the development of critical slowing down of Eu spin fluctuations over a relatively wide critical region $\left(T-T_{\mathrm{N}}\right) / T_{\mathrm{N}}<5$ above $T_{\mathrm{N}}$. We also found from ${ }^{29} \mathrm{Si} \mathrm{NMR}$ that the spin fluctuations are strongly suppressed by magnetic field in the paramagnetic state above $20 \mathrm{~K}$. These characteristic spin dynamics observed over a wide region of temperature and magnetic field suggest the presence of magnetic frustration in the spin system. Such frustration would underlie the mechanism stabilizing the short-period skyrmion lattice observed in this compound.
\end{abstract}

DOI: 10.1103/PhysRevB.104.045145

\section{INTRODUCTION}

The rare-earth compound EuPtSi crystallizes in a noncentrosymmetric chiral structure (cubic LaIrSi type) belonging to the same space group $P 2{ }_{1} 3$ as $\mathrm{MnSi}$ and other B20 materials, where the divalent Eu ions form a three-dimensional lattice of corner-sharing triangles called the trillium lattice [1-4]. This compound recently drew considerable attention as a system having a unique magnetic skyrmion phase characterized by an extremely short magnetic modulation period $(\sim 18 \AA)$ [5,6], an order of magnitude shorter than that of $\mathrm{MnSi}(\sim 180 \AA)$ [7]. The skyrmion lattice is further characterized by strong magnetic anisotropy, suggesting strong coupling of the skyrmion lattice with the crystal lattice $[4-6,8,9]$.

In noncentrosymmetric chiral magnets such as $\mathrm{MnSi}$, the formation of the skyrmion lattice has been attributed to the competition between ferromagnetic (FM) exchange and the Dzyaloshinskii-Moriya (DM) interactions resulting from the spin-orbit coupling and the lack of inversion symmetry [10]. This mechanism is, however, not simply applicable for EuPtSi, since the DM interaction is unexpected for $\mathrm{Eu}^{2+}$ ions which do not have orbital angular momentum. Instead, magnetic frustration from the geometry of the trillium lattice, and/or competing magnetic correlations, have been proposed to play a major role in stabilizing the short-period skyrmion lattice [11].

\footnotetext{
*Present address: Department of Quantum Matter, AdSM, Hiroshima University, Higashihiroshima, Hiroshima 739-8530, Japan.
}

In zero magnetic field, EuPtSi exhibits a helimagnetic phase transition at $T_{\mathrm{N}}=4 \mathrm{~K}$ [1-4]. This phase transition is first order in nature, and furthermore, signatures of developing magnetic fluctuations as precursors to the transition have been reported. In the specific heat, for example, a sharp spike appears at $T_{\mathrm{N}}$, on the top of a broad hump occurring below at $\sim 7 \mathrm{~K}$ [2]. Magnetic entropy at $T_{\mathrm{N}}$ is only half the full $R \ln 8$ expected for the 7/2 spin of $\mathrm{Eu}^{2+}$. Eventually, it reaches the full value around $30 \mathrm{~K}[2,8]$. Similarly, the magnetic susceptibility exhibits a sudden drop at $T_{\mathrm{N}}$, followed by an inflection point in the Curie-Weiss behavior around $7 \mathrm{~K}$ [12], and the linewidth in the ${ }^{151} \mathrm{Eu}$ Mössbauer spectrum shows a gradual upturn below $8 \mathrm{~K}$ [13]. From these results, the possibility of a fluctuation-induced, first-order transition has been discussed for EuPtSi, which is further supported by the recent observation of a tricritcal point [12]. Interestingly, the fluctuation-induced first-order transition has also been suggested to occur in other B20 helimagnets, such as $\mathrm{MnSi}$ and $\mathrm{Cu}_{2} \mathrm{OSeO}_{3}$ [14-16].

In this paper, we report the results of muon spin relaxation $(\mu \mathrm{SR})$ and ${ }^{29} \mathrm{Si}$ NMR measurements performed on EuPtSi. At zero magnetic field (ZF), the critical slowing down of $\mathrm{Eu}$ spin fluctuations has been detected in a relatively wide temperature region $\left(T-T_{\mathrm{N}}\right) / T_{\mathrm{N}}<5$ above $T_{\mathrm{N}}$. We also found from the NMR measurements that the spin fluctuations are rapidly suppressed by applied magnetic field in the paramagnetic region above $20 \mathrm{~K}$. Our observations strongly support the fluctuation-induced, first-order transition mechanism, and further suggest the presence of magnetic frustration in the spin system. 


\section{EXPERIMENT}

Single crystals of EuPtSi were grown by the Bridgman method. Details of the sample preparation are described in Ref. [4]. For NMR studies, we prepared ${ }^{29} \mathrm{Si}$ isotope-enriched ( $\sim 99 \%)$ single crystals, in order to enhance the ${ }^{29} \mathrm{Si}$ NMR signal intensity. The natural abundance of the ${ }^{29} \mathrm{Si}$ is only $4.7 \%$.

Time-differential $\mu$ SR measurements were performed in the ZF/longitudinal-field configuration [17] using the D1 $\mu$ SR spectrometer of the Japan Proton Accelerator Research Complex (J-PARC) for the temperature range $15<T<300 \mathrm{~K}$ and the GPS instrument of the Paul Scherrer Institut (PSI) for $1.6<T<15 \mathrm{~K}$ with a conventional ${ }^{4} \mathrm{He}$ cryostat. The bin size of time-to-digital converters (TDCs) was set to $8 \mathrm{~ns}$ at J-PARC and $391 \mathrm{ps}$ at PSI, limiting the time resolution of positron-counting systems. Single crystals cut along the (111) plane were used for the experiment at J-PARC, while a polycrystalline sample of larger volume was used for the experiment at PSI. Spin-polarized surface $\mu^{+}$beams were incident to the samples, with the initial muon spin polarization direction along the $\langle 111\rangle$ direction at J-PARC. We confirmed that the results are compatible between the J-PARC and PSI experiments; the temperature dependencies of the muon relaxation rate obtained at the two facilities are smoothly connected in the paramagnetic state around $15 \mathrm{~K}$.

${ }^{29} \mathrm{Si}$ NMR measurements were carried out on a powdered single crystal sample using a superconducting magnet and a phase-coherent, pulsed spectrometer. The ${ }^{29} \mathrm{Si}$ nuclei have the nuclear gyromagnetic ratio $\gamma_{\mathrm{n}} / 2 \pi=8.4578 \mathrm{MHz} / \mathrm{T}$ and $I=$ $1 / 2$. The NMR spectrum was obtained by sweeping frequency at fixed external field and integrating the spin-echo signal intensity. The spin-lattice relaxation rate $1 / T_{1}$ was measured using the saturation-recovery method.

\section{EXPERIMENTAL RESULTS}

\section{A. Zero-field $\mu$ SR}

$\mu \mathrm{SR}$ and NMR are both powerful techniques, allowing us to investigate the microscopic properties of electron spin dynamics with different, but complementary time windows of observation. NMR is more sensitive to slow spin dynamics. However, the high sensitivity often provides an extremely short nuclear relaxation near a magnetic phase transition, preventing the observation of an NMR signal in a wide temperature range near the phase transition. On the other hand, $\mu$ SR can detect relaxation times shorter than $0.1 \mu \mathrm{s}$, about two orders of magnitude shorter than the NMR capability; it thus can be more suitable for tracking slow spin dynamics emerging near the phase transition. A further advantage of $\mu \mathrm{SR}$ is that one can measure the spin dynamics without applying a magnetic field.

Figure 1 shows time-differential $\mu$ SR spectra obtained under $\mathrm{ZF}$ at various temperatures above and below $T_{\mathrm{N}}$. The muon decay asymmetries were all well described by the simple exponential depolarization function, $A(t)=A_{0} \exp \left(-t / T_{1, \mu}\right)$, where $A_{0}$ is the effective initial asymmetry and $1 / T_{1, \mu}$ is the muon spin relaxation rate. Such a single exponential character for the relaxation function is generally expected when the

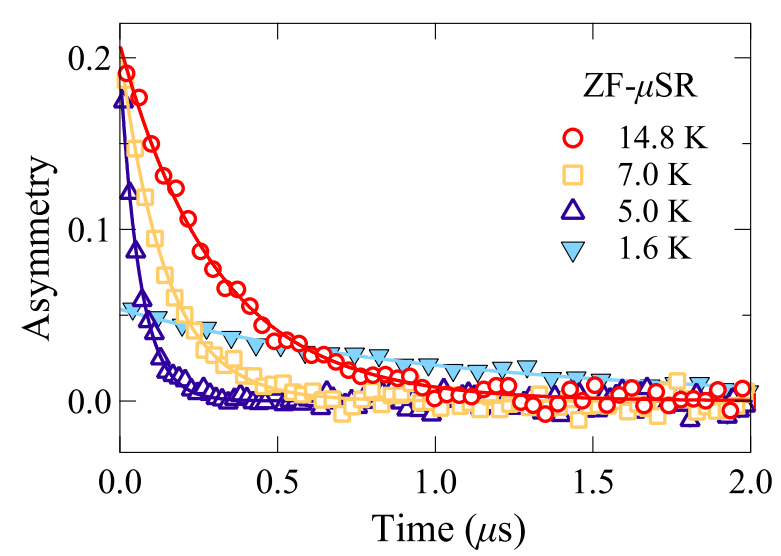

FIG. 1. ZF- $\mu$ SR spectra at $14.8,7.0$, and $5.0 \mathrm{~K}$ in the paramagnetic phase (open symbols) and at $1.6 \mathrm{~K}$ in the helimagnetic phase (filled triangle). The solid lines are the best fits to a single exponential function: $A(t)=A_{0} \exp \left(-t / T_{1, \mu}\right)$.

relaxation is dynamical in origin; here it would be dominated by the fluctuations of giant Eu spin moments $\left(\sim 7 \mu_{\mathrm{B}}\right)$.

Figure 2 shows the temperature dependence of $1 / T_{1, \mu}$. With decreasing temperature, $1 / T_{1, \mu}$ exhibits a rapid increase below about $20 \mathrm{~K}$, followed by a distinct peak just at $T_{\mathrm{N}} \sim$ $4 \mathrm{~K}$. This behavior is characteristic of a magnetic phase transition accompanied by a critical slowing down. Below $T_{\mathrm{N}}, 1 / T_{1, \mu}$ is sharply suppressed, and at the same time, $A_{0}$ is also discontinuously suppressed to about one -third of its original value, as shown in the inset of Fig. 2. This behavior for $A_{0}$ is fairly consistent with magnetic ordering in a polycrystalline sample where the direction of the local fields is completely randomized. In such a case, the $\mu$ SR signal splits into one-third longitudinal and two-thirds transverse components with respect to the initial muon spin direction [18]. The longitudinal part corresponds to one-third of the local field components along the initial spin direction, which effectively act as longitudinal fields for muon spins. The relaxation rate of this component, $1 / T_{1, \mu}$, reflects the nature of low-energy magnetic excitations in the ordered state. The remaining

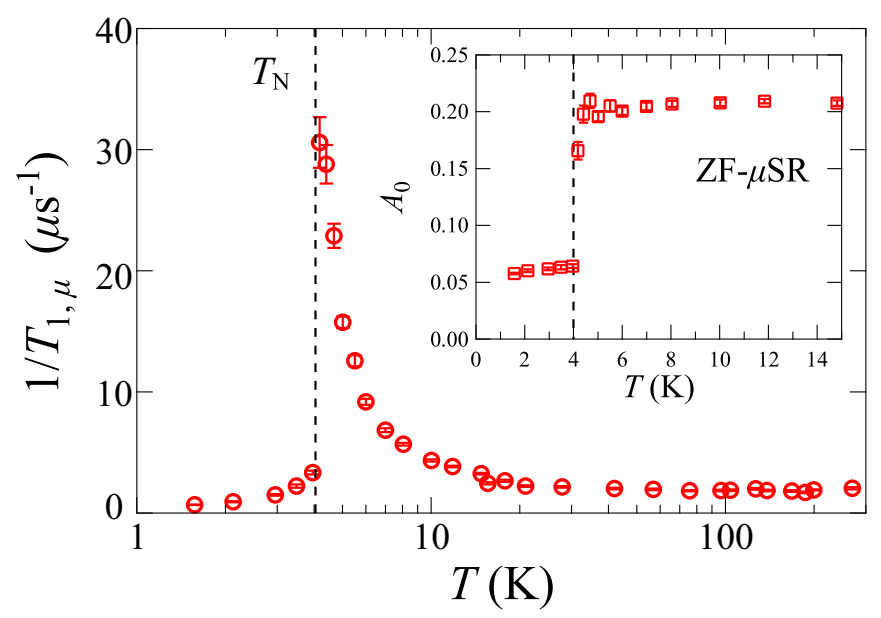

FIG. 2. Temperature dependence of the relaxation rate $1 / T_{1, \mu}$ in ZF. The inset shows the temperature dependence of the effective initial asymmetry $A_{0}$ for the data obtained at PSI. 


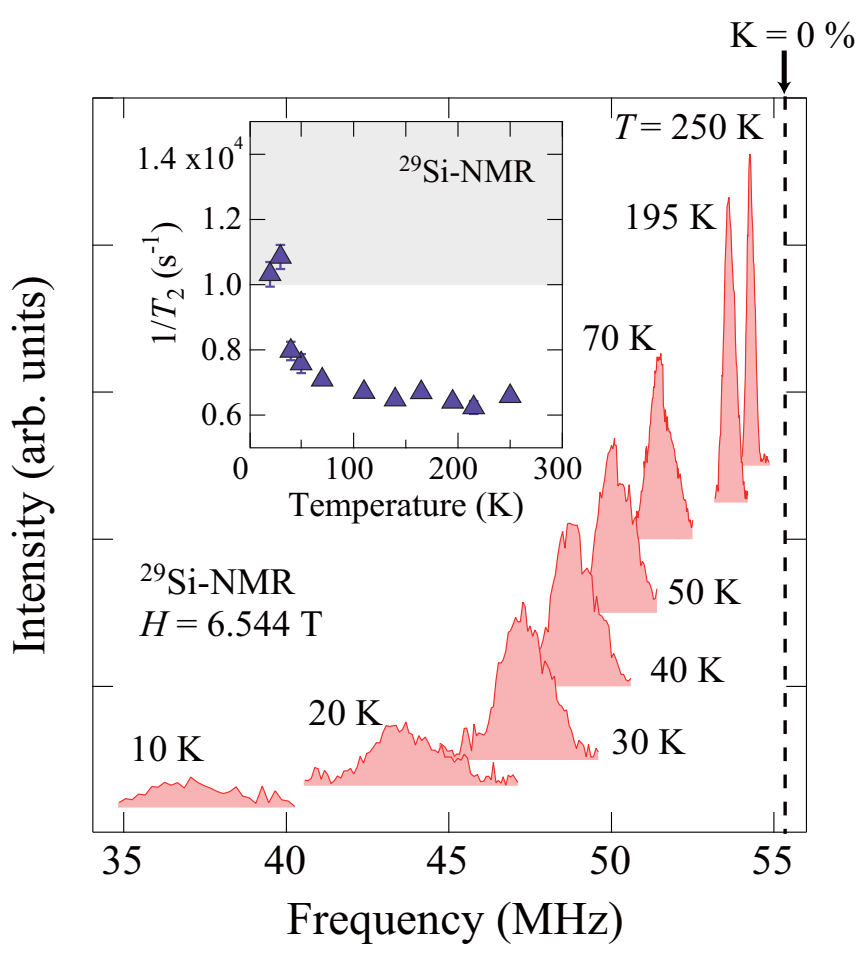

FIG. $3 .{ }^{29} \mathrm{Si}$ NMR spectra in the magnetic field $H=6.544 \mathrm{~T}$ at various temperatures. The inset shows the temperature dependence of $1 / T_{2}$.

transverse part generally has a damped cosine structure corresponding to muon spin precession in effective transverse fields. In our case, the cosine signal was averaged out or promptly damped to zero because of large internal fields at muon sites and it was not clearly captured in our configuration with the TDC bin size of 391 ps. This is in line with the roughly estimated value of a field spread at muon sites, $\sim 1.4 \mathrm{~T}$, which is calculated for the $7 \mu_{\mathrm{B}}$ ordered moment using a typical coupling constant of $0.2 \mathrm{~T} / \mu_{\mathrm{B}}$ for $f$-electron compounds [19]. The corresponding damping rate, $1.2 \times 10^{9} \mathrm{~s}^{-1}$, is close to the resolution limit.

Note that neutron diffraction studies of EuPtSi have found a second magnetic transition from an incommensurate to a commensurate structure at $T_{\mathrm{N}}^{*} \sim 2.5 \mathrm{~K}$ [5]. In the present $\mu \mathrm{SR}$ study, however, we could not resolve any distinct anomaly in either $A_{0}$ or $1 / T_{1, \mu}$ in the vicinity of $T_{\mathrm{N}}^{*}$ (Fig. 2).

\section{B. ${ }^{29}$ Si NMR}

NMR probes the local environment of the observed nucleus and its dynamics. The crystallographic position of the nucleus is usually well defined, so that the results can be straightforwardly compared with theories. In addition, we can conduct most NMR experiments in a small-scale laboratory, without using a large experimental facility. This advantage allowed us to investigate detailed field dependence of magnetic properties in the present study.

Figure 3 shows the temperature dependence of the ${ }^{29} \mathrm{Si}$ NMR spectrum measured at the fix field of $H=6.544 \mathrm{~T}$. Since ${ }^{29} \mathrm{Si}$ has a nuclear spin of $1 / 2$, and there is only one crystallographic Si site per unit cell, a single, sharp NMR peak

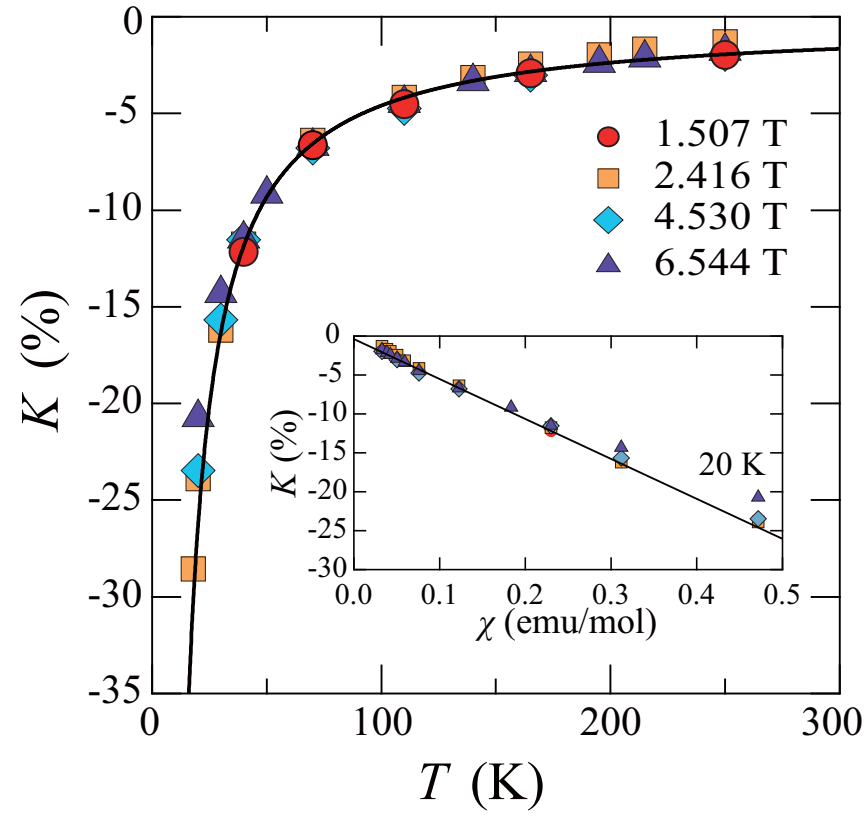

FIG. 4. Temperature dependence of the Knight shift at various magnetic fields in the paramagnetic state. The solid line is the result of the Curie-Weiss fit with the Weiss temperature $\theta_{\mathrm{P}}=4.0 \mathrm{~K}$. The inset shows $K(T)$ versus the magnetic susceptibility $\chi(T)$ with temperature as an implicit parameter. The slope of the solid line yields hyperfine coupling constant $A_{\mathrm{hf}}^{s}=-2.87 \mathrm{kOe} / \mu_{\mathrm{B}}$.

has been observed at higher temperatures. With decreasing temperature, the spectrum broadens, and moves on to lower frequencies due to negative Knight shifts $(K)$. The linewidth increases roughly proportional to $|K|$, indicating that the distribution of $K$ is its major origin.

With further decrease of temperature, NMR signal intensity decreases significantly below about $20 \mathrm{~K}$, and is completely wiped out below $10 \mathrm{~K}$. The loss of the signal intensity is attributed to extremely fast transverse relaxation of the nuclei (i.e., to a short $T_{2}$ ) on the timescale of the NMR window (see inset to Fig. 3). It is thus associated with the critical slowing down of Eu moment fluctuations detected by $\mu \mathrm{SR}$ in the same temperature region. Note that the loss of NMR signal intensity during a magnetic phase transition is not a rare observation. It usually occurs in a very narrow temperature region near the transition temperature. The signal intensity is generally recovered in the ordered state, after static spin order is well established. In the present study, however, we could not detect an NMR signal even at temperatures well below $T_{\mathrm{N}}(\sim 100 \mathrm{mK})$. The latter result suggests continuing, slow Eu spin fluctuations in the ordered state, as was also indicated by $\mu \mathrm{SR}$ data. An extremely broad distribution of internal magnetic fields can be another possible source of the loss of the NMR signal intensity in the helical ordered state.

Figure 4 shows the temperature dependence of $K(T)$ measured in several different magnetic fields. Here, $K$ is determined by the peak position of the NMR spectrum at each temperature. The solid line indicates a fit with the Curie-Weiss law $K(T)=C /\left(T-\theta_{\mathrm{P}}\right)$, which yields $\theta_{\mathrm{P}}=4 \mathrm{~K} . K$ does not show any field dependence in the entire range of temperatures and fields. 


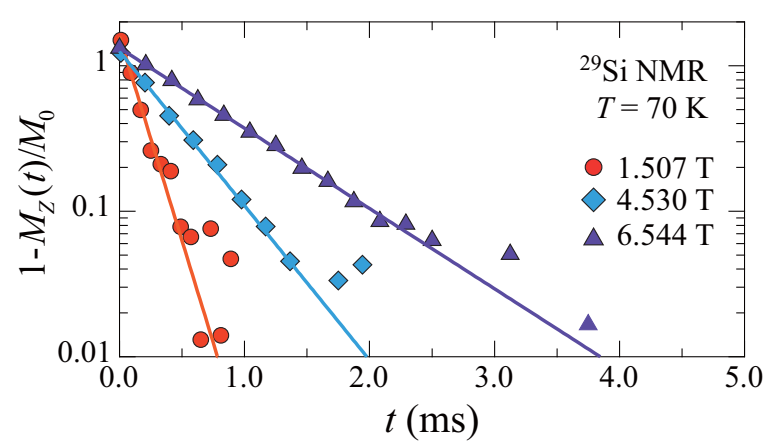

FIG. 5. Examples of ${ }^{29} \mathrm{Si}$ nuclear magnetization recovery measured at $T=70 \mathrm{~K}$ in $H=1.507,4.530$, and $6.544 \mathrm{~T}$. The solid lines are fits with a single exponential function $1-M_{z}(t) / M_{0}=$ $\exp \left(-t / T_{1}\right)$.

For $f$-electron systems the $K$ of ligand nuclei is generally composed of a spin part $K^{\mathrm{s}}$ and a Van Vleck part $K^{\mathrm{v}}$. These components are related to a $T$-dependent spin susceptibility $\chi^{\mathrm{s}}$ and a $T$-independent Van Vleck susceptibility $\chi^{\mathrm{V}}$ as follows:

$$
\begin{aligned}
K(T) & =K^{\mathrm{s}}(T)+K^{\mathrm{v}} \\
& =\frac{1}{N_{\mathrm{A}} \mu_{\mathrm{B}}}\left[A_{\mathrm{hf}}^{\mathrm{s}} \chi^{\mathrm{s}}(T)+A_{\mathrm{hf}}^{\mathrm{v}} \chi^{\mathrm{v}}\right],
\end{aligned}
$$

where $A_{\mathrm{hf}}^{\mathrm{s}}\left(A_{\mathrm{hf}}^{\mathrm{v}}\right)$ is the spin (Van Vleck) hyperfine (hf) coupling constant, $N_{\mathrm{A}}$ is Avogadro's number, and $\mu_{\mathrm{B}}$ is the Bohr magneton. In the inset to Fig. 4, we plot $K(T)$ against $\chi(T)$ with temperature as an implicit variable. $K(T)$ maintains a good linear relation with $\chi(T)$, and thus the slope of the $K$ vs $\chi$ plot yields the spin part of the hyperfine coupling constants $A_{\mathrm{hf}}^{\mathrm{s}}=-2.87 \pm 0.07 \mathrm{kOe} / \mu_{\mathrm{B}}$. On the other hand, the intercept of the line at $\chi=0$ is nearly zero. In EuPtSi, susceptibility measurements indicate that $\chi^{\mathrm{V}} \simeq 0$, and hence, $K^{\mathrm{v}}$ should be $\simeq 0$, as is observed. Note that a negative value for $A_{\mathrm{hf}}^{\mathrm{s}}$ at ligand nuclei has been reported for several Eu-based intermetallic compounds, such as $\mathrm{EuGa}_{4}$ [20], $\mathrm{EuAl}_{4}$ [21,22], $\mathrm{EuCo}_{2} \mathrm{P}_{2}$ [23], and $\mathrm{EuCo}_{2} \mathrm{As}_{2}$ [24]. The negative $A_{\mathrm{hf}}^{\mathrm{s}}$ suggests the dominance of the Ruderman-Kittel-Kasuya-Yosida coupling mechanism [21].

The spin-lattice relaxation rate $1 / T_{1}$ has also been measured in several different magnetic fields. Figure 5 shows examples of ${ }^{29} \mathrm{Si}$ nuclear magnetization recoveries, measured at $T=70 \mathrm{~K}$ in $H=1.507,4.530$, and $6.544 \mathrm{~T}$, respectively. In all measurements, the nuclear recoveries were well fitted with a single exponential function $1-M_{z}(t) / M_{0}=$ $\exp \left(-t / T_{1}\right)$, as expected for $I=1 / 2$ nuclei, and thus a unique $T_{1}$ value was obtained at each temperature and field. The single $T_{1}$ component in a powder sample indicates a $T_{1}$ process dominated by isotropic spin fluctuations.

In general, $T_{1}$ is related to the low-energy spin-fluctuation densities perpendicular to the quantization axis [25]. The observed temperature and field dependence of $1 / T_{1} T$ are shown in Fig. 6. In the same figure, we also plot the temperature dependence of $1 / T_{1, \mu} T$, together with the bulk susceptibility $\chi(T)$. We found that $1 / T_{1, \mu} T$ scales linearly with $\chi(T)$ in the entire paramagnetic region above $20 \mathrm{~K}$, while their temperature dependencies distinctly deviate from each other in the

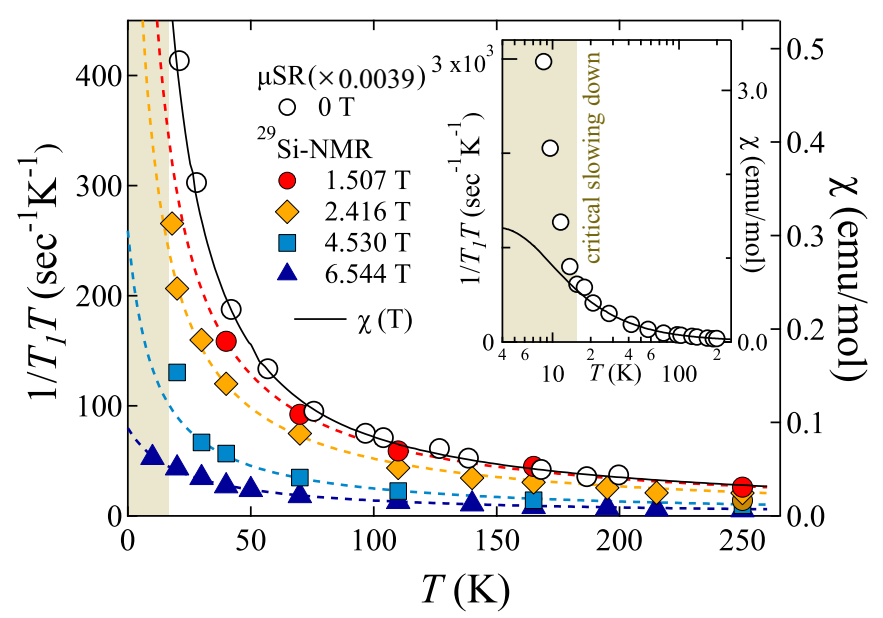

FIG. 6. The temperature and field dependencies of the NMR $1 / T_{1} T$. The broken lines indicate the results of a CW fit to the $1 / T_{1} T$ data above $25 \mathrm{~K}$, yielding values of the Weiss temperature $\theta_{\mathrm{P}}$ and the Curie constant $C$ plotted in Fig. 7. In the same figure and the inset, we also plot the temperature dependencies of $1 / T_{1, \mu} T$ and $\chi(T)$. The $1 / T_{1, \mu} T$ values are multiplied by a factor 0.0039 for scaling with the $\chi(T)$ and NMR $1 / T_{1} T$ data.

critical region below $20 \mathrm{~K}$ (see the inset of Fig. 6). The $1 / T_{1} T$ measured by NMR at a lower field of $1.5 \mathrm{~T}$ exhibits a similar temperature dependence to that of $1 / T_{1, \mu} T$, and thus of $\chi(T)$. With increasing applied magnetic field, however, $1 / T_{1} T$ is suppressed rapidly over a wide temperature region. The origin of this suppression is not very obvious, but here we tentatively analyze the behavior by fitting the data with the Curie-Weiss (CW) law above $25 \mathrm{~K}$. The broken lines in Fig. 6 show the results of the $\mathrm{CW}$ fit, which give the Weiss temperature $\theta_{\mathrm{P}}$ and the Curie constant $C$ shown in Fig. 7. Both the $\theta_{\mathrm{P}}$ and the $C$ exhibit strong field dependence, decreasing linearly with increasing applied magnetic field. Interestingly, we note that $\theta_{\mathrm{P}}$ changes sign from positive to negative at $H \sim 1.2 \mathrm{~T}$, where the formation of the skyrmion lattice has been observed at low temperatures [3-6].
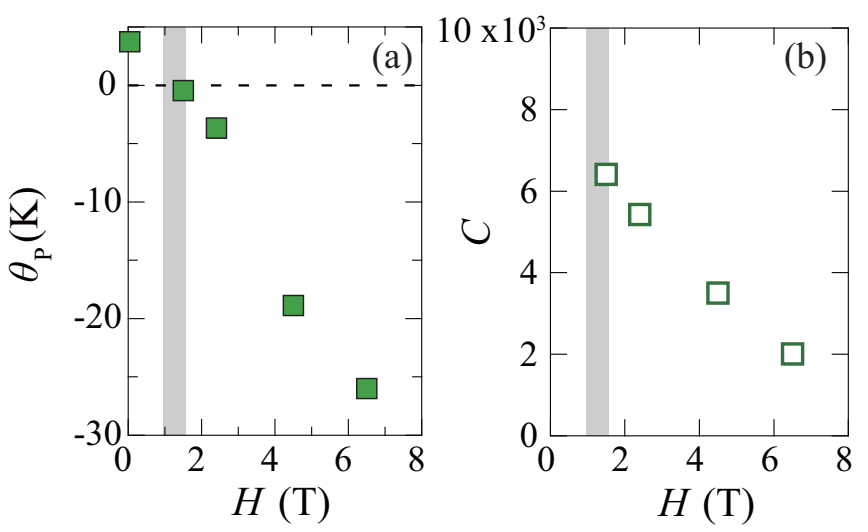

FIG. 7. Field dependence of (a) the Weiss temperature $\theta_{\mathrm{P}}$ and (b) the Curie constant $C$ extracted from $\mathrm{CW}$ fits to the $1 / T_{1} T$ and $1 / T_{1, \mu} T(H=0)$ data. The gray area corresponds to the field region where the formation of the skyrmion lattice has been observed. 


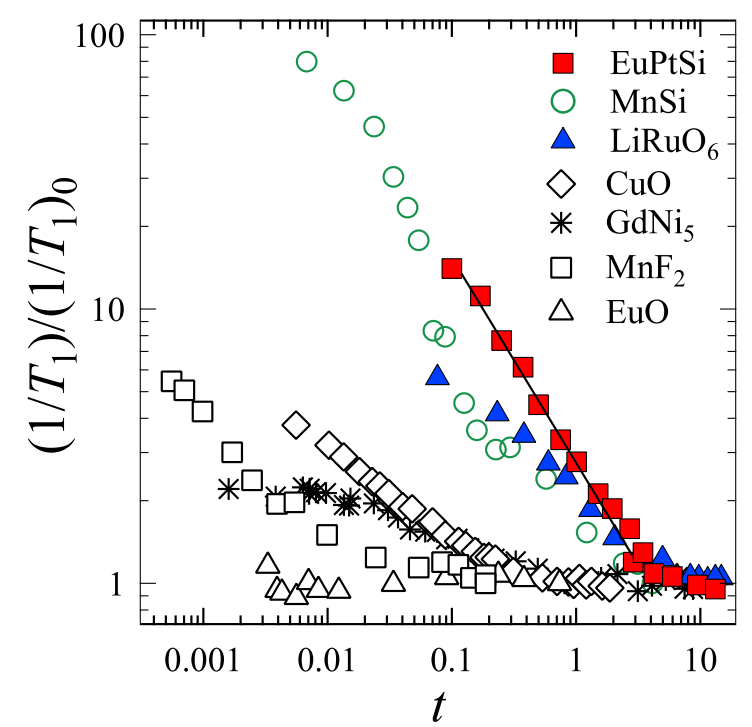

FIG. 8. Log-log plots of the normalized relaxation rate $\left(1 / T_{1}\right) /$ $\left(1 / T_{1}\right)_{0}$ against the reduced temperature $t=\left(T-T_{\mathrm{N}, \mathrm{C}}\right) / T_{\mathrm{N}, \mathrm{C}}$ for $\mathrm{Eu}-$ $\mathrm{PtSi}$, and several magnets, $\mathrm{GdNi}_{5}$ [29], $\mathrm{MnF}_{2}$ [30], $\mathrm{EuO}$ [31], MnSi [32] (from $\mu \mathrm{SR}$ ), $\mathrm{CuO}$ [28], and $\mathrm{Li}_{7} \mathrm{RuO}_{6}$ [33] (from NMR), where $\left(1 / T_{1}\right)_{0}$ is the high temperature limit of $1 / T_{1}$. The data in EuPtSi follows Eq. (2) in a temperature range of $t<5$ with the exponent $\omega=0.74$, as shown by the solid line.

\section{DISCUSSION}

\section{A. Critical slowing down}

In a magnetic system, when the temperature is decreased in the paramagnetic state toward the ordering temperature, the paramagnetic spins form into clusters and exhibit a critical slowing down in a critical temperature region. In the present study, the critical slowing down of Eu spin moments has been observed by $\mu \mathrm{SR}$ as a strong upturn of $1 / T_{1, \mu}$ below about $20 \mathrm{~K}$. It has been also detected by NMR as the wipeout of the signal intensity in the same temperature region.

In the critical temperature region, one can expect a temperature dependence of the relaxation rate,

$$
1 / T_{1} \propto t^{-\omega}
$$

where $t=\left(T-T_{\mathrm{N}, \mathrm{C}}\right) / T_{\mathrm{N}, \mathrm{C}}$ is the reduced temperature, and the $\omega$ is a critical exponent. In Fig. 8, we plot in the log-log scale the normalized relaxation rate $\left(1 / T_{1}\right) /\left(1 / T_{1}\right)_{0}$ against $t$ for several magnets, where $\left(1 / T_{1}\right)_{0}$ is the high temperature limit of $1 / T_{1}$. For EuPtSi, $1 / T_{1, \mu}$ follows Eq. (2) in a relatively wide temperature range of $t<5$ with the exponent $\omega=0.74$, as shown by the solid line.

The critical region of $t<5$ observed in EuPtSi is a signature of magnetic frustration in the spin system. In general, for simple magnets, critical slowing down occurs only in a narrow temperature region near a transition temperature, typically in $t \ll 1[26,27]$. As examples of this, we plot in Fig. $8\left(1 / T_{1}\right) /\left(1 / T_{1}\right)_{0}$ for $\mathrm{CuO}$ [28], $\mathrm{GdNi}_{5}$ [29], $\mathrm{MnF}_{2}$ [30], and $\mathrm{EuO}[31]$; all of these magnets possess a narrow critical region. On the other hand, frustrated magnets often exhibit a wide critical region, e.g., $\mathrm{MnSi}(t<4)$ [32] and $\mathrm{Li}_{7} \mathrm{RuO}_{6}$ $(t<7)$ [33]; the latter is a Heisenberg antiferromagnet with a triangular lattice, and thus has geometric frustration. The critical region of $t<5$ is also wider than $t<1$ observed for the two-dimensional square lattice spin systems [33-36].

The critical exponent $\omega$ observed here is a composite of dynamical and static critical exponents. The dynamical scaling theory gives $\omega=v(z+2-d-\eta)$ in the usual notation. For the three-dimensional ( $d=3$ ) Heisenberg model, one can expect the dynamical exponent $z=2.5$ and 1.5 for FM and antiferro- (AF) magnets, respectively [26,30,37]. Then, using static exponents $v=0.705$ and $\eta=0.033$ for the Heisenberg model [38], one can obtain $\omega=1.03$ and 0.33 for the FM and AF cases, respectively. The observed value $\omega=0.74$ in EuPtSi thus does not match with these two simple cases, implying a rather complex nature for the fluctuations.

Note that, while the critical fluctuations are at the origin of scaling laws close to second-order phase transitions, if the low-energy fluctuations are too abundant, the system may evade the critical point to avoid the large entropy associated with the fluctuations by undergoing a first-order phase transition [39]. As mentioned, the possibility of such a fluctuation-induced, first-order transition has been recently discussed for EuPtSi [2,12]. Theoretically, if an order parameter possesses a large number of components $N \geqslant 4$, its self-interaction with critical fluctuations is already sufficient to drive the transition first order [40]. Regarding EuPtSi, the lattice-symmetry operations on the observed propagation vector of the helical order yields 12 distinct ordering vectors; the number of order parameter components is thus large enough to satisfy the condition [12]. Experimentally, on the other hand, while the first-order nature of the transition has been well established [12], the presence of strong fluctuations above $T_{\mathrm{N}}$ had only been indirectly suggested. Here, by means of the $\mu \mathrm{SR}$ and NMR, we have successfully observed the enhanced critical fluctuations in a wide temperature region above $T_{\mathrm{N}}$. This further supports the scenario of the fluctuation-induced, first-order transition in EuPtSi.

\section{B. Paramagnetic fluctuations}

In the paramagnetic state, the static spin susceptibility $\chi(T)$ of EuPtSi follows a $\mathrm{CW}$ law with a positive Weiss temperature $\theta_{\mathrm{P}}=5-10 \mathrm{~K}$ [1-3]. Then, magnetic correlations have been considered to be predominantly ferromagnetic. This has also been confirmed by our NMR Knight shift, providing $\theta_{\mathrm{P}} \sim 4 \mathrm{~K}$. For the dynamical spin susceptibility, on the other hand, the situation is found to be rather complex. At zero field, the $1 / T_{1, \mu} T$ scales linearly to $\chi(T)$ above $20 \mathrm{~K}$, suggesting the dynamical spin susceptibility to be dominated by FM fluctuations with the wave vector $\boldsymbol{q}=(0,0,0)$. However, the NMR $1 / T_{1} T$ demonstrates that the fluctuation behaviors are strongly field dependent, being suppressed with increasing magnetic field, where $\theta_{\mathrm{P}}$ changes the sign from positive to negative value. This result implies the occurrence of a field-induced crossover from ferromagnetically to antiferromagnetically dominated fluctuations around $H \sim 1.2 \mathrm{~T}$.

In NMR studies, a suppression of $1 / T_{1} T$ in magnetic fields is generally observed in localized ferromagnets. In these materials, FM correlations are developing among local moments, so that low-energy spin fluctuations are enhanced rapidly as the temperature decreases. Because of the ferromagnetically enhanced susceptibility, however, an applied 
field quickly quenches out these fluctuations, resulting in progressive suppression of $1 / T_{1} T$. In such a case, however, any sharp anomalies associated with the transition, including the critical slowing down, should be smeared out under magnetic fields, since applied magnetic field forces a change of the FM phase transition into a crossover; this is obviously not the case for EuPtSi.

The observed $1 / T_{1} T$ behaviors would be described most naturally by introducing the competing magnetic correlations, that is, $1 / T_{1}$ responds to magnetic fluctuations enhanced at both the FM $\boldsymbol{q}=(0,0,0)$ and the propagation vector of the incommensurate helical state, $\boldsymbol{q}=(0.2,0.3, \delta)(\delta \simeq 0.04)$ [5]. The latter drives the critical slowing down toward the helimagnetic transition. Thus, since the FM component will be suppressed more rapidly under magnetic field, we can expect a field-induced crossover for the predominant fluctuations. Such competing magnetic correlations will naturally explain a relatively lower $T_{\mathrm{N}}$ for EuPtSi, compared with other Eubased magnets having a magnetic transition typically around $15-100 \mathrm{~K}$. The competing magnetic correlations further explain the wide critical regions $(t<5)$ and the unusual critical exponent $(\omega=0.74)$ observed in this study.

It is also important to note that EuPtSi possesses a geometric frustration inherent to the trillium lattice. Theoretically, the degree of geometric frustration on this lattice is shown to lie on the border between a frustrated degenerate ground state and an ordered state [41-43]. Within the mean-field approximation, the AF Heisenberg model yields a degenerate ground state, where the degenerate wave vectors $\boldsymbol{q}$ form a spheroidlike surface centered at $\boldsymbol{q}=(0,0,0)$ [42]. Such a degeneracy will naturally enhance low-energy fluctuations at zero field, but the fluctuations will be suppressed sharply under magnetic field, since the applied field lifts the degeneracy by breaking the high symmetry of the cubic lattice [12]. Geometric frustration can thus be another possible source of strongly field-dependent fluctuations. Further experimental efforts, such as NMR under uniaxial stress, are needed to understand this issue in detail.

\section{SUMMARY}

Using $\mu \mathrm{SR}$ and ${ }^{29} \mathrm{Si}$ NMR techniques complementarily, we have investigated the dynamical properties of Eu spin moments in the paramagnetic state of EuPtSi. The experiments revealed characteristic spin dynamics that develop over a wide temperature and magnetic field region, which suggest the presence of frustration in the spin system, resulting from competing magnetic correlation and/or the geometrically frustrated trillium lattice. It is expected that these frustrations underlie the mechanism stabilizing the unique skyrmion lattice with a short modulation period and strong anisotropy found in EuPtSi.

\section{ACKNOWLEDGMENTS}

The authors would like to thank T. Matsumura and R. E. Walstedt for the critical reading of the manuscript. The $\mu$ SR experiments at J-PARC and PSI were performed under the user programs J-PARC Proposal No. 2018A0089 and PSI Proposal No. 20190228. Part of the work was supported by the Japan Society for the Promotion of Science KAKENHI: J-Physics (Grants No. JP15K21732 and No. JP15H05884) and Grants-in-Aid for Scientific Research (B) and (C) (Grants No. 20H01864 and No. 20K12484).
[1] D. T. Adroja, B. D. Padalia, S. K. Malik, R. Nagarajan, and R. Vijayaraghavan, J. Magn. Magn. Mater. 89, 375 (1990).

[2] D. G. Franco, Y. Prots, C. Geibel, and S. Seiro, Phys. Rev. B 96, 014401 (2017).

[3] M. Kakihana, K. Nishimura, Y. Ashitomi, T. Yara, D. Aoki, A. Nakamura, F. Honda, M. Nakashima, Y. Amako, Y. Uwatoko, T. Sakakibara, S. Nakamura, T. Takeuchi, Y. Haga, E. Yamamoto, H. Harima, M. Hedo, T. Nakama, and Y. Ōnuki, J. Electron. Mater. 46, 3572 (2017).

[4] M. Kakihana, D. Aoki, A. Nakamura, F. Honda, M. Nakashima, Y. Amako, S. Nakamura, T. Sakakibara, M. Hedo, T. Nakama, and Y. Ōnuki, J. Phys. Soc. Jpn. 87, 023701 (2018).

[5] K. Kaneko, M. D. Frontzek, M. Matsuda, A. Nakao, K. Munakata, T. Ohhara, M. Kakihana, Y. Haga, M. Hedo, T. Nakama, and Y. Ōnuki, J. Phys. Soc. Jpn. 88, 013702 (2019).

[6] C. Tabata, T. Matsumura, H. Nakao, S. Michimura, M. Kakihana, T. Inami, K. Kaneko, M. Hedo, T. Nakama, and Y. Ōnuki, J. Phys. Soc. Jpn. 88, 093704 (2019).

[7] Y. Ishikawa and M. Arai, J. Phys. Soc. Jpn. 53, 2726 (1984).

[8] T. Takeuchi, M. Kakihana, M. Hedo, T. Nakama, and Y. Ōnuki, J. Phys. Soc. Jpn. 88, 053703 (2019).

[9] T. Takeuchi, M. Kakihana, M. Hedo, T. Nakama, and Y. Ōnuki, J. Phys. Soc. Jpn. 89, 093703 (2020).

[10] N. Nagaosa and Y. Tokura, Nat. Nanotechnol. 8, 899 (2013).
[11] T. Okubo, S. Chung, and H. Kawamura, Phys. Rev. Lett. 108, 017206 (2012).

[12] T. Sakakibara, S. Nakamura, S. Kittaka, M. Kakihana, M. Hedo, T. Nakama, and Y. Ōnuki, J. Phys. Soc. Jpn. 88, 093701 (2019).

[13] Y. Homma, M. Kakihana, Y. Tokunaga, M. Yogi, M. Nakashima, A. Nakamura, Y. Shimizu, D. Li, A. Maurya, Y. J. Sato, F. Honda, D. Aoki, Y. Amako, M. Hedo, T. Nakama, and Y. Ōnuki, J. Phys. Soc. Jpn. 88, 094702 (2019).

[14] A. Bauer, M. Garst, and C. Pfleiderer, Phys. Rev. Lett. 110, 177207 (2013).

[15] M. Janoschek, M. Garst, A. Bauer, P. Krautscheid, R. Georgii, P. Böni, and C. Pfleiderer, Phys. Rev. B 87, 134407 (2013).

[16] H. C. Chauhan, B. Kumar, J. K. Tiwari, and S. Ghosh, Phys. Rev. B 100, 165143 (2019).

[17] A. Amato, H. Luetkens, K. Sedlak, A. Stoykov, R. Scheuermann, M. Elender, and A. Raselli, Rev. Sci. Instrum. 88, 093301 (2017).

[18] A. Yaouanc and P. Dalmas de Réotier: Muon Spin Rotation, Relaxation, and Resonance: Applications to Condensed Matter, 1st ed. (Oxford University Press, Oxford, UK, 2011).

[19] A. Amato, Rev. Mod. Phys. 69, 1119 (1997).

[20] M. Yogi, S. Nakamura, N. Higa, H. Niki, Y. Hirose, Y. Ōnuki, and H. Harima, J. Phys. Soc. Jpn. 82, 103701 (2013). 
[21] A. M. van Diepen, K. H. J. Buschow, and H. W. de Wijn, J. Chem. Phys. 51, 5259 (1969).

[22] H. Niki, S. Nakamura, N. Higa, H. Kuroshima, T. Toji, M. Yogi, A. Nakamura, M. Hedo, T. Nakama, Y. Ōnuki, and H. Harima, J. Phys.: Conf. Ser. 592, 012030 (2015).

[23] N. Higa, Q.-P. Ding, M. Yogi, N. S. Sangeetha, M. Hedo, T. Nakama, Y. Ōnuki, D. C. Johnston, and Y. Furukawa, Phys. Rev. B 96, 024405 (2017).

[24] Q.-P. Ding, N. Higa, N. S. Sangeetha, D. C. Johnston, and Y. Furukawa, Phys. Rev. B 95, 184404 (2017).

[25] T. Moriya, Prog. Theor. Phys. 16, 641 (1956).

[26] C. Hohenemser, N. Rosov, and A. Kleinhammes, Hyperfine Interact. 49, 267 (1989).

[27] S. W. Lovesey and E. Engdahl, J. Phys.: Condens. Malter 7, 769 (1995).

[28] Y. Itoh, T. Imai, T. Shimizu, T. Tsuda, H. Yasuoka, and Y. Ueda, J. Phys. Soc. Jpn. 59, 1143 (1990).

[29] A. Yaouanc, P. Dalmas de Réotier, P. C. M. Gubbens, A. M. Mulders, F. E. Kayzel, and J. J. M. Franse, Phys. Rev. B 53, 350 (1996).

[30] M. Attenborough, I. Hall, O. Nikolov, S. R. Brown, and S. F. J. Cox, Hyperfine Interact. 108, 423 (1997).
[31] S. J. Blundell, T. Lancaster, F. L. Pratt, P. J. Baker, W. Hayes, J.P. Ansermet, and A. Comment, Phys. Rev. B 81, 092407 (2010).

[32] R. Kadono, T. Matsuzaki, T. Yamazaki, S. R. Kreitzman, and J. H. Brewer, Phys. Rev. B 42, 6515 (1990).

[33] Y. Itoh, C. Michioka, K. Yoshimura, K. Nakajima, and H. Sato, J. Phys. Soc. Jpn. 78, 023705 (2009).

[34] T. Imai, C. P. Slichter, K. Yoshimura, and K. Kosuge, Phys. Rev. Lett. 70, 1002 (1993).

[35] C. Bucci and G. Guidi, Phys. Rev. B 9, 3053 (1974).

[36] E. P. Maarschall, A. C. Botterman, S. Vega, and A. R. Miedema, Physica 41, 473 (1969).

[37] B. I. Halperin and P. C. Hohenberg, Phys. Rev. 177, 952 (1969).

[38] J. C. Le Guillou and J. Zinn-Justin, Phys. Rev. B 21, 3976 (1980).

[39] S. A. Brazovskii, Sov. Phys. JETP 41, 85 (1975).

[40] P. Bak, S. Krinsky, and D. Mukamel, Phys. Rev. Lett. 36, 52 (1976).

[41] T. E. Redpath and J. M. Hopkinson, Phys. Rev. B 82, 014410 (2010).

[42] J. M. Hopkinson and H.-Y. Kee, Phys. Rev. B 74, 224441 (2006).

[43] S. V. Isakov, J. M. Hopkinson, and H.-Y. Kee, Phys. Rev. B 78, 014404 (2008). 